\title{
Successful treatment of two Japanese ESRD cases with severe COVID-19 pneumonia
}

\author{
Tatsuki Abe $^{1}$ (1) $\cdot$ Takehiro Izumo $^{2} \cdot$ Akihiro Ueda $^{3} \cdot$ Munehiro Hayashi $^{4} \cdot$ Yoshitaka Ishibashi $^{1}$
}

Received: 17 May 2020 / Accepted: 16 July 2020 / Published online: 26 July 2020

(c) Japanese Society of Nephrology 2020

\begin{abstract}
Novel coronavirus disease 2019 (COVID-19) is a highly infectious and deadly disease, spreading worldwide. There are limited data about the clinical course of end-stage renal disease (ESRD) patients infected with COVID-19. However, previous cohort studies showed a high mortality rate of ESRD patients infected with COVID-19. We report here two Japanese ESRD patients confirmed with severe COVID-19 pneumonia. Case 1 was a 60-year-old man with ESRD due to diabetic nephropathy who were infected with COVID-19 and exhibited acute respiratory distress syndrome (ARDS) requiring mechanical ventilation and intensive care unit (ICU) admission. He was treated with tocilizumab and intravenous immunoglobulin (IVIG). After 6 days of treatment in ICU, he was extubated. Case 2 was a 68 -year-old woman undergoing maintenance hemodialysis for 17 years who also exhibited ARDS due to COVID-19. Her clinical course resembles case 1. Our experience of these two cases indicates that anti-cytokine therapy might be effective for severe COVID-19 pneumonia in ESRD patients.
\end{abstract}

Keywords Acute respiratory distress syndrome (ARDS) - Coronavirus disease 2019 (COVID-19) · Diabetic nephropathy · End-stage renal disease (ESRD) $\cdot$ Intravenous immunoglobulin (IVIG) $\cdot$ Tocilizumab

\section{Background}

Since December 2019, the current outbreak of novel coronavirus disease 2019 (COVID-19) has spread to many countries including Japan. Some previous studies revealed the clinical characteristics of patients infected with COVID-19 [1]. However, there are limited reports about the clinical course of end-stage renal disease (ESRD) patients infected with COVID-19. We report here 2 Japanese patients with ESRD, who recovered from severe COVID-19 pneumonia.

Tatsuki Abe

tatsuki.abe0601@gmail.com

1 Department of Nephrology, Japanese Red Cross Medical Center, 4-1-22 Hiro-o, Shibuya-Ku, Tokyo 150-8935, Japan

2 Department of Respiratory Medicine, Japanese Red Cross Medical Center, 4-1-22 Hiro-o, Shibuya-Ku, Tokyo 150-8935, Japan

3 Department of Infectious Diseases, Japanese Red Cross Medical Center, 4-1-22 Hiro-o, Shibuya-Ku, Tokyo 150-8935, Japan

4 Department of Emergency and Critical Care Medicine, Japanese Red Cross Medical Center, 4-1-22 Hiro-o, Shibuya-Ku, Tokyo 150-8935, Japan

\section{Case reports}

\section{Case 1}

A 60-year-old man with ESRD due to diabetic kidney disease (DKD) presented with 4 days of cough, slight fever and fatigue. On admission, his temperature was $37.7{ }^{\circ} \mathrm{C}$ with an oxygen saturation of $98 \%$ in room air. Laboratory tests revealed an increased level of blood urea nitrogen, creatinine, CRP, ferritin, soluble IL-2 receptor and D-dimer, and decreased lymphocyte count (Table 1). A chest CT scan showed bilateral and peripheral ground-glass opacities (GGO) in the lung (Fig. 1a). A nasopharyngeal swab for RT-PCR was positive for COVID-19, he was diagnosed as COVID-19 pneumonia. Then, he was treated with ceftriaxone, azithromycin, peramivir and favipiravir. Hemodialysis (HD) was started on day 3. His pneumonia was gradually getting worse after the first session of HD. His body temperature rose above $38{ }^{\circ} \mathrm{C}$ and his oxygen requirements increased day by day. A CT scan showed the area of GGO expanded on day 4 (Fig. 1a). Given his hyper-inflammatory status with elevated IL-6 $(47.8 \mathrm{pg} / \mathrm{mL})$, the patient was administered $8 \mathrm{mg} / \mathrm{kg}$ of tocilizumab twice and $2500 \mathrm{mg}$ of intravenous immunoglobulin (IVIG) twice. No concomitant 
Table 1 Laboratory tests of case 1 and case 2 on admission

\begin{tabular}{lll}
\hline & Case 1 & Case 2 \\
\hline AST (U/L) & 17 & 24 \\
ALT (U/L) & 18 & 13 \\
LDH (U/L) & 207 & 325 \\
ALP (U/L) & 208 & 510 \\
$\gamma$ GTP (U/L) & 18 & 30 \\
Creatine kinase (U/L) & 185 & 58 \\
Total bilirubin (mg/dL) & 0.6 & 0.3 \\
Total protein $(\mathrm{g} / \mathrm{dL})$ & 6.8 & 6.5 \\
Albumin $(\mathrm{g} / \mathrm{dL})$ & 3.7 & 3 \\
Blood urea nitrogen $(\mathrm{mg} / \mathrm{dL})$ & 91 & 29 \\
Creatinine $(\mathrm{mg} / \mathrm{dL})$ & 10.04 & 4.69 \\
Uric acid $(\mathrm{mg} / \mathrm{dL})$ & 6.7 & 5.5 \\
Sodium $(\mathrm{mg} / \mathrm{dL})$ & 133 & 137 \\
Potassium $(\mathrm{mg} / \mathrm{dL})$ & 4.2 & 5 \\
Chloride $(\mathrm{mg} / \mathrm{dL})$ & 94 & 96 \\
Calcium $(\mathrm{mg} / \mathrm{dL})$ & 8 & 7.5 \\
Phosphorus $(\mathrm{mg} / \mathrm{dL})$ & 7.2 & 5.5 \\
Ferritin $(\mathrm{ng} / \mathrm{mL})$ & 834 & 193 \\
Soluble IL-2 receptor $(\mathrm{U} / \mathrm{mL})$ & 1805 & 1883 \\
D-dimer $(\mu \mathrm{g} / \mathrm{mL})$ & 3.2 & 9.7 \\
White blood cell $(/ \mathrm{mL})$ & 4770 & 6190 \\
Neutrocyte $(/ \mathrm{mL})$ & 3720 & 5680 \\
Lymphocyte $(/ \mathrm{mL})$ & 530 & 520 \\
Hemoglobin $(\mathrm{g} / \mathrm{dL})$ & 10.3 & 12.9 \\
Platelet $(/ \mathrm{mL})$ & 14.4 & \\
\hline & & 12.7 \\
\hline
\end{tabular}

drugs such as antihistamine agents were used with tocilizumab and IVIG. On day 6, he exhibited acute respiratory distress syndrome (ARDS) with $\mathrm{PaO}_{2} / \mathrm{FiO}_{2}$ ratio of 133 , and he was transferred to the intensive care unit (ICU) and intubated. During 6 days of treatment in ICU, his fever fell below $37{ }^{\circ} \mathrm{C}$ on day 7 , his hemodynamic status was stable, $\mathrm{PaO}_{2} / \mathrm{FiO}_{2}$ ratio was gradually improved to 310 on day 9 , and the level of CRP decreased (Fig. 1b), suggesting that his pneumonia was improved. He was extubated on day 12 . Because of the negative results of RT-PCR assay, he was released from the isolation unit on day 29. He didn't recover from incident hemodialysis.

\section{Case 2}

A 68-year-old woman on HD for 17 years due to DKD was referred to our emergency department with a chief complaint of fever, cough, and diarrhea. Her body temperature was $38.6{ }^{\circ} \mathrm{C}$ with an oxygen saturation of $96 \%$ in room air. Laboratory tests revealed similar results as with case 1 (Table 1 ). A nasopharyngeal swab for RT-PCR was positive for COVID-19. Her clinical course after admission resembles that of case 1 . She was treated with azithromycin, meropenem, and peramivir immediately. On day 3 , her pneumonia was rapidly getting worse. Her oxygen requirements acutely increased to $6 \mathrm{~L} / \mathrm{min}$ via a non-rebreather mask with $\mathrm{PaO}_{2}$ of 140 in the night, meaning she developed ARDS. On day 4, she was intubated and treated in an intensive care unit with
Fig. 1 a Chest computed tomographic images of case 1. CT scan showed the area of bilateral and peripheral groundgrass opacities (GGO) rapidly increased on day 4 , compared to day 1 . On day 18 , the GGO almost disappeared. b Clinical course of case 1 . The level of CRP decreased and $\mathrm{PaO}_{2} / \mathrm{FiO}_{2}$ ratio was improved after the usage of tocilizumab and IVIG (a)

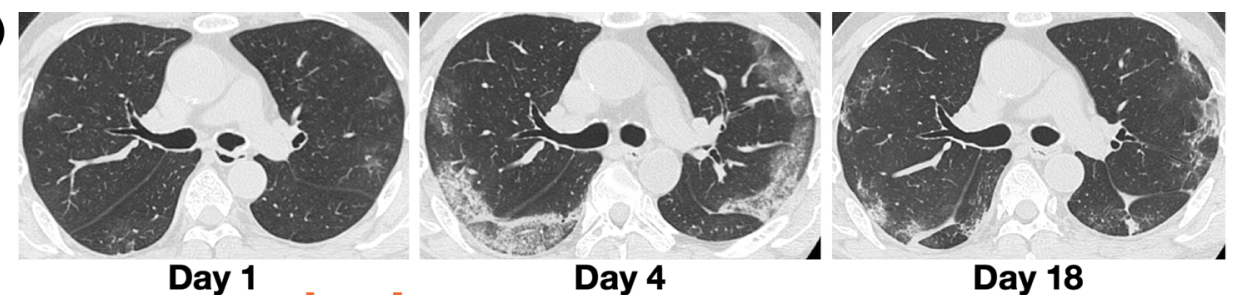

(b)

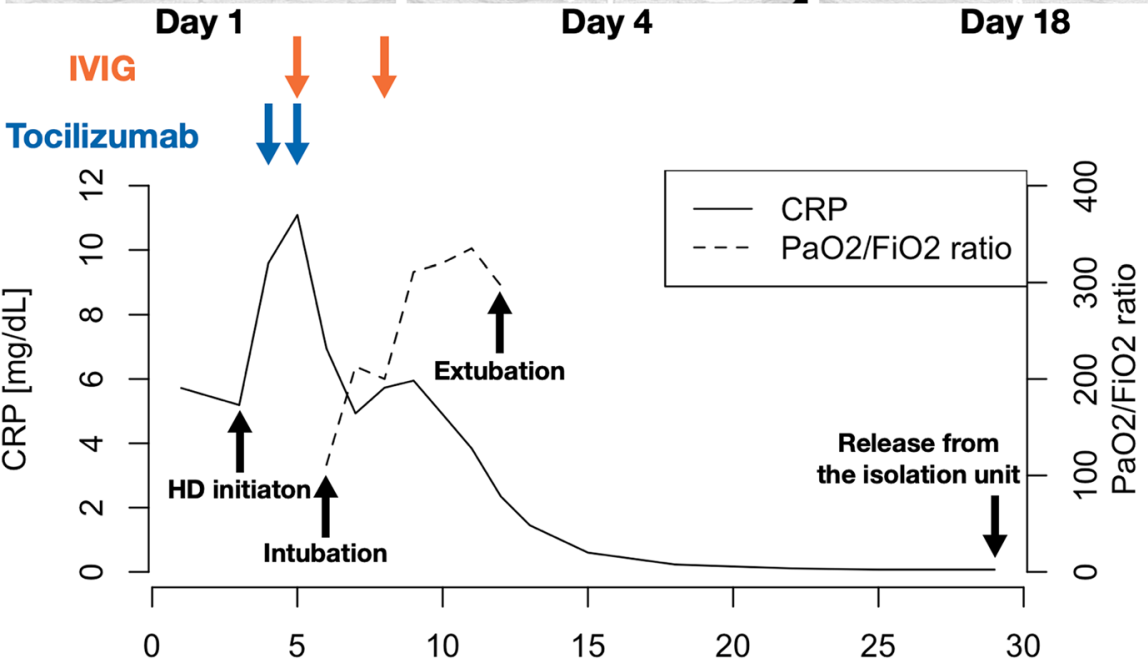

hospital days 
Fig. 2 a A chest computed tomographic image and chest $\mathrm{X}$-rays of case 2. A CT scan showed bilateral and peripheral ground-grass opacities (GGO) on day 1 . The GGO on X-rays expanded especially through day 1 to day 7 . On day 15 , the area of GGO was reduced. b Clinical course of case 2 . As with case 1 , the level of CRP decreased and $\mathrm{PaO}_{2} / \mathrm{FiO}_{2}$ ratio was improved after the administration of tocilizumab and IVIG (a)

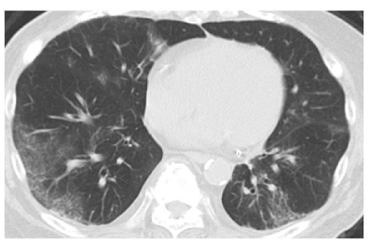

Day 1

(b)

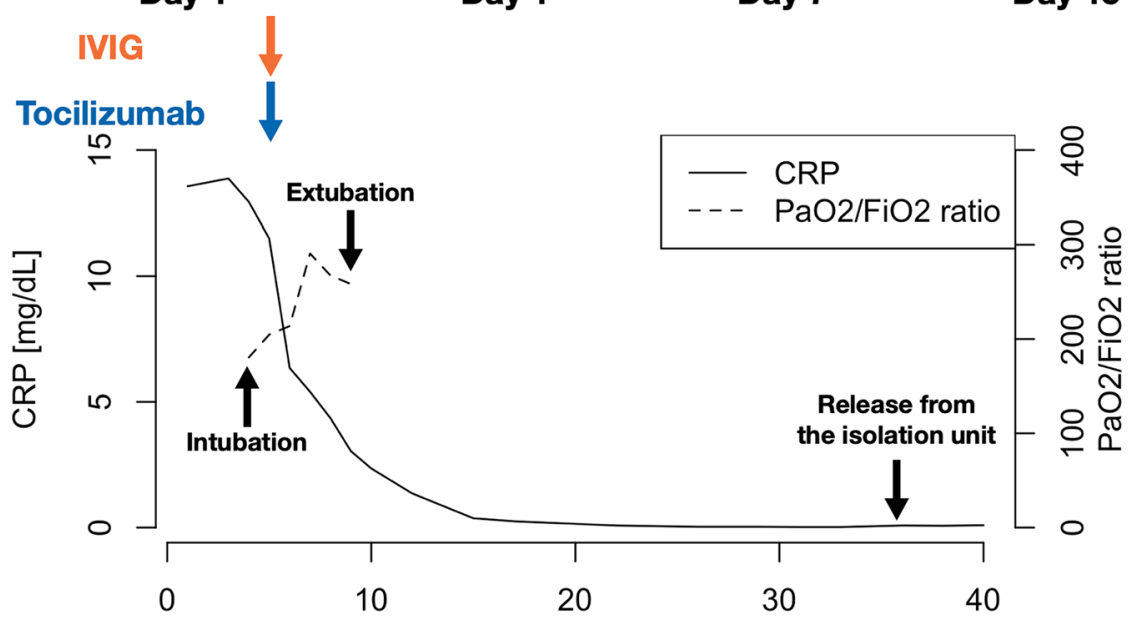

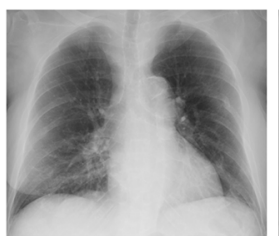

Day 1

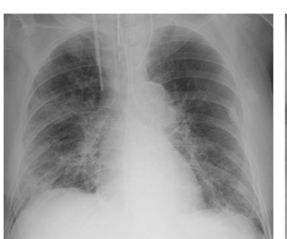

Day 7

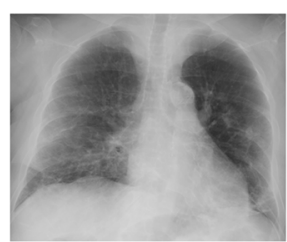

Day 15 hospital days mechanical ventilation. Favipiravir, $8 \mathrm{mg} / \mathrm{kg}$ of tocilizumab and $2500 \mathrm{mg}$ of IVIG was added to treat her COVID-19 pneumonia. Before administration of tocilizumab, the level of IL-6 was $93.6 \mathrm{pg} / \mathrm{mL}$. Shortly after treatment with these drugs, her body temperature dropped, her breathing status improved with her $\mathrm{PaO}_{2} / \mathrm{FiO}_{2}$ ratio of 267 on day 8 , and her CRP reduced (Fig. 2b). Finally, she was extubated and returned from ICU on day 9. She was released from the isolation unit on day 36 based on negative results of PCR assays.

\section{Discussion}

We have experienced two Japanese ESRD patients who recovered from severe COVID-19 pneumonia. This is the first time to report in detail the clinical course of ESRD patients, who recovered from severe COVID-19 pneumonia. Our patients had diabetes and hypertension, risk factors for severity [1]. Previous cohort studies showed ESRD was also related to aggravation [2, 3]. Trujillo et al. reported 25 patients undergoing maintenance HD, who were hospitalized because of COVID-19. In the study, 10 patients $(40 \%)$ exhibited ARDS and 7 patients $(28 \%)$ died. Similarly, Alberici et al. reported 21 patients with COVID-19 receiving HD, 5 of whom (24\%) died. These studies showed a high mortality rate in ESRD patients. In contrast, our patients recovered from a critical condition such as ARDS requiring ICU admission and mechanical ventilation. The reason for the recovery from severe COVID-19 pneumonia could be early usage of tocilizumab and IVIG. As Alberci et al. reported, a biphasic therapy may be effective for COVID-19. In the first phase, the patients are asymptomatic or present with flu-like symptoms. Antiviral therapy including favipiravir would be a therapeutic option. In the second phase, the patients exhibited a hyperinflammatory status called cytokine storm. Therefore, immunosuppressive therapy might be therapeutic options. Tocilizumab, a humanized monoclonal antibody against IL-6 receptor, and IVIG were reported to be options $[4,5]$. We used these drugs quickly when the patients exhibited ARDS. These drugs might exert a protective effect against the development of ARDS. In fact, after administration of these drugs, C-reactive protein started to decrease to normal range in 2 weeks (Fig. 1b). It coincided with the recovery from ARDS. Therefore, early treatment may lead to improved survival even for ESRD patients.

In conclusion, we described two ESRD patients, who fully recovered from severe COVID-19 pneumonia. The recovery might be attributed to the usage of tocilizumab and IVIG in the early phase of progressive disease.

Acknowledgements We thank both patients and all involved doctors. 


\section{Compliance with ethical standards}

Conflict of interest All the authors have declared no conflict of interest.

Ethical approval All procedures performed in studies involving human participants were in accordance with the ethical standards of the institutional and/or national research committee and with the 1964 Helsinki Declaration and its later amendments or comparable ethical standards.

Informed consent Informed consent was obtained from all individual participants included in the study.

\section{References}

1. Guan WJ, Ni ZY, Hu Y, et al. Clinical characteristics of coronavirus disease 2019 in China. N Engl J Med. 2020;382(18):1708-20.

2. Trujillo H, Caravaca-Fontán F, Sevillano Á, et al. SARS-CoV-2 infection in hospitalized patients with kidney disease. Kidney Int Rep. 2020;5(6):905-9.
3. Alberici F, Delbarba E, Manenti C, et al. Management of patients on dialysis and with kidney transplant during SARSCOV-2 (COVID-19) pandemic in Brescia, Italy. Kidney Int Rep. 2020;5(5):580-5.

4. Toniati P, Piva S, Cattalini M, et al. Tocilizumab for the treatment of severe COVID-19 pneumonia with hyperinflammatory syndrome and acute respiratory failure: a single center study of 100 patients in Brescia, Italy. Autoimmun Rev. 2020;19(7):102568.

5. Prete M, Favoino E, Catacchio G, Racanelli V, Perosa F. SARSCoV-2 infection complicated by inflammatory syndrome. Could high-dose human immunoglobulin for intravenous use (IVIG) be beneficial? Autoimmun Rev. 2020;19(7):102559.

Publisher's Note Springer Nature remains neutral with regard to jurisdictional claims in published maps and institutional affiliations. 